\title{
Cardiotoxicity induced by antineoplastic drug Daunorubicin and its amelioration: A review of literature
}

\author{
Zorawar $\mathrm{S}^{*}$ and Harmanpreet $\mathrm{K}$ \\ Department of Zoology, Khalsa College Amritsar, Punjab, India
}

\begin{abstract}
Daunorubicin (DNR) belongs to anthracycline class of drugs that has been reported with a significant role in treating leukemia. But use of DNR is restricted due to associated cardiotoxicity. In this review, we present the cardiotoxic effects associated with DNR along with some ameliorative agents. DNR induced sub chronic cardiomyopathy, down regulation of cytokines and stem cell markers which may reflect impaired chemotaxis, migration and homing of stem cells; and tissue repair in the heart. DNR may affect the heart by forming hydrophobic interactions and hydrogen bonds with cardiac myosin. DNR cardiotoxicity may change with age of patient as systematic clearance of drug decreases with age. Cardiotoxicity effects may include inhibition of cardioprotective epoxyeicosatrienoic acid. Adenosine, sodium ferulate and pomegranate have been reported as promising cardio-protectants against DNR induced cardiotoxicity. GnRH conjugates containing DNR also showed no associated cytotoxic effects on cardiomyocytes. Future research on amelioration of DNR associated toxicity is highly recommended.
\end{abstract}

\section{Introduction}

Anthracycline is a type of antibiotic that comes from Streptomyces peucetius bacteria. Each anthracycline drug contains rubicin as suffix in its name, which is derived from Latin word rubidus, that means red. Anthracyclines are aromatic polyketides available in large variety of forms because of structural differences in the aglycone [1]. The generic name anthracycline was given by $\mathrm{H}$. Brockmann to a group of glycosidic compounds whose aglycones, called anthracyclinones, had a basic structure of 7,8,9,10-Tetrahydro 5,12-naphthacene quinone [1]. The common anthracycline drugs include daunorubicin, doxorubicin, epirubicin and idarubicin. Important functional characteristics of the anthracycline antibiotics are (a) the planar anthraquinone ring system (b) quinone groups on the unsaturated rings (c) stereochemistry of the D ring substitution at position 9 and (d) the amino sugar, daunosamine, which provides water solubility and chemical architecture for stabilizing DNA binding [2].

Anthracyclines are used to treat many types of cancer. These are effective against broad range of malignancies including solid and hematological tumors. These are also used against leukemia, lymphoma, breast cancer, bladder cancer, uterine cancer, bowel cancer and other broad-spectrum therapies which make them first-line chemotherapeutic drugs [1]. Anthracyclines are known to inhibit the synthesis of DNA and RNA by intercalation and base modification, thus preventing the replication of rapidly growing cancer cells [3]. These interact with topoisomerase II enzyme which results in disturbance in the replication and transcription processes [3]. The mechanism may involve the production of iron-mediated free oxygen radicals that damage the DNA and cell membranes (Figure 1) [3].

\section{Daunorubicin}

The first anthracycline drug identified was DNR from the soil bacterium Streptomyces peucetius. DNR was found effective in treatment of leukemia and lymphomas in 1960's [4]. It was the first anthracycline drug analog to be characterized structurally and sterochemically [1].

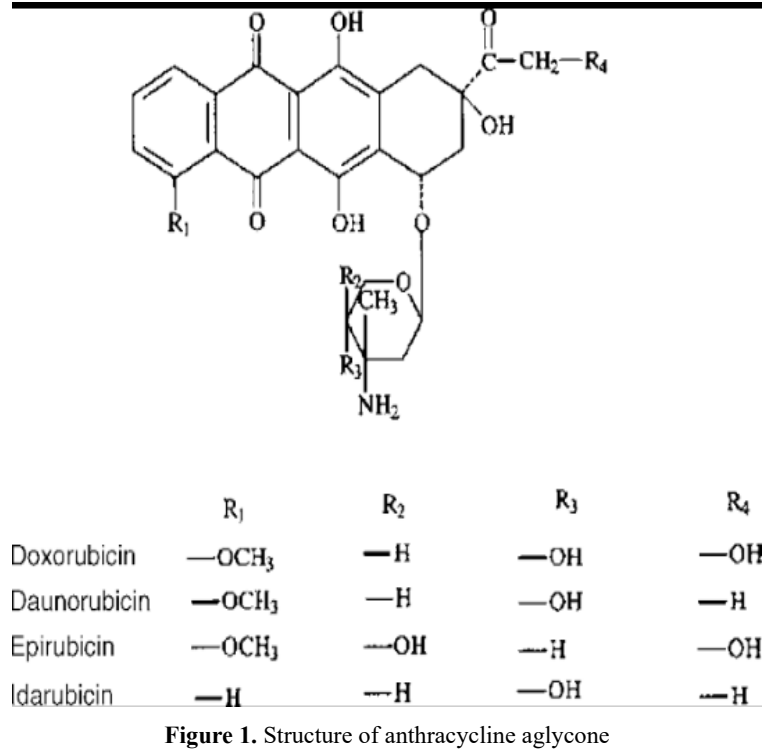

DNR drug is available both in the form of solution and powder which is given intravenously to the patients along with other chemotherapeutic medications [5]. The dose and schedule are determined by the person's size and the treatment regimen being used. It can be administered alone or in combination with other drugs [6]. DNR is injected once a day, when it is used to treat the acute myeloid leukemia (AML) and once a

${ }^{\star}$ Correspondence to: Zorawar S, Department of Zoology, Khalsa College Amritsar, Punjab, India, Tel: +91-9417230075; E-mail: zorawarsinghs@rediffmail.com

Key words: daunorubicin, cardiotoxicity, cardiac myosin, cytotoxic effects, cardiomyocytes, amelioration, cardio-protectants

Received: March 04, 2019; Accepted: March 15, 2019; Published: March 18, 2019 
week in case of acute lymphocytic leukemia [5]. Urine color of patient turns pink or red due to introduction of DNR. It is not blood, but due to renal clearance of the drug from the patient's body [6]. DNR can act during multiple phases of the cell cycle and is considered cell-cycle specific [7].

DNR is beneficial in the treatment of leukemia, but its use is restricted due to associated cardiotoxic effects. Decrease in cardiac function in AML patient's after treatment with DNR in combination with cytarabine can be determined by drop in left ventricular ejection [8]. DNR may interfere with the pumping action of heart or may lower the body's ability to make blood cells. Thus, patient may need blood transfusion leading to bleeding complications and infections [7]. An early biomarker of DNR-induced cardiotoxicity is troponin and its level may be taken as directly proportional to the amount of cardiac damage. Troponin is an intracellular enzyme which can be detected in the blood when cells have lysed and expulsed their contents. In case of DNR toxicity, a rise in troponin may indicate cardiomyocyte apoptosis as well as myofibril degradation [9]. Heart damage may occur during the treatment or after many months or years [5]. A patient can receive only up to a certain amount of DNR during his lifetime. The 'lifetime maximum dose' may be lesser if patient has heart risk factors like old age, radiation to chest and use of other toxic medications [7]. Another side effect of DNR may include leaking out of DNR from administering line into patient's body which can cause damage to the muscles and skin in contact. DNR is a vesicant that may cause extensive tissue damage and blistering if it escapes from the vein. Administered DNR may cause burning sensation through the arm of patient and discoloration around the administering site (Table 1) [7].

Several studies have been retrieved which demonstrated different effects of DNR on heart. Different models including rat and human have been used. In a study, male Wistar rats were treated with DNR to induce acute cardiomyopathy $(6 \times 3 \mathrm{mg} / \mathrm{kg}$, i.p., every $48 \mathrm{hr}$, DAU-A) or subchronic cardiomyopathy (15 mg/kg, i.v., DAU-C). In this experiment, reduced body weight, decrease in left ventricular weight and elevated Nppa, Nppb and Myh7 (isomyosins) levels were observed in both groups, but Myh6 decreased only in DAU-C group. Up-regulated gp91phox and down-regulated Abcb8 were also found only in DAU-C group. Experiment also showed decreased expressions of Scf and Vegf (cytokines), as well as of stem cell markers. All these effects caused down-regulation of cytokines and stem cell markers which reflected impaired chemotaxis, migration and homing of stem cells; and tissue repair in the heart in sub-chronic but not acute model of DAU cardiomyopathy [10]. In another study, the heart samples were collected from donors with and without Down syndrome. These samples were used to investigate the determinants for anthracyclinerelated cardiotoxicity including cardiac daunorubicin reductase activity (DA), carbonyl reductase (CR)/ aldo-keto reductase (AKRs) protein expression, mitochondrial DNA content (mtDNA), and AKR7A2 DNA methylation status. Clinical evidences suggested that patients with leukemia and Down syndrome were at increased risk for anthracyclinerelated cardiotoxicity. CR and AKRs catalyze the reduction of DNR and doxorubicin into cardiotoxic C-13 alcohol metabolites. Studies revealed that cardiac mtDNA content, mtDNA (4977) deletion frequency, and AKR7A2 protein content are the most important variables in determining DNR reductase activity [11,12].

Introduction of chemotherapeutic drug targeting techniques potentially increased the tumor selectivity of drugs and decreased their cardiotoxicity. Increased expression of gonadotropin-releasing hormone $(\mathrm{GnRH})$ receptors on the surface of tumor cells has been reported. Sixteen different GnRH- conjugates containing doxorubicin, DNR and methotrexate were developed and investigated. Their cytotoxicity was determined on primary human cardiac myocytes (HCM) and human umbilical vein endothelial cells (HUVEC). As a result, anticancer drug-GnRH-based conjugates with no cytotoxic effect on cardiomyocytes were found. In the future, these compounds could provide a better targeted antitumor therapy without any cardiotoxic adverse effects [13]. Myocardial cells were used to investigate the dose and time-dependent cellular enzyme release induced by either Adriamycin or DNR primary cultures in rat. Myocardial cells with exposure to DNR or Adriamycin (24 hour) at concentration of 1.8 or 18

Table 1. Studies related to cardiotoxicity of daunorubicin

\begin{tabular}{|c|c|c|c|c|c|}
\hline Sr. No. & AUTHORS & YEAR & ANIMAL & RESULTS & REFERENCE NO. \\
\hline 1 & Srankova et al. & 2016 & Rat & $\begin{array}{l}\text { Down-regulation of cytokines and stem cell markers may reflect impaired chemo- } \\
\text { taxis, migration and homing of stem cells and tissue repair in the heart in subchronic } \\
\text { but not acute model of DNR cardiomyopathy }\end{array}$ & [10] \\
\hline 2 & Hoefer et al. & 2016 & Human & $\begin{array}{l}\text { The most relevant variables for the synthesis of cardiotoxic daunorubicinol are cardiac } \\
\text { mtDNA content, mtDNA ( } 4977 \text { ) deletion frequency, and AKR7A2 protein content }\end{array}$ & [11] \\
\hline 3 & Lian et al. & 2015 & Rat & $\begin{array}{l}\text { Treatment with sodium ferulate inhibits the decrease in heart rates induced by DNR ( } p \\
<0.05) \text {, increases the left ventricular end diastolic pressure }\end{array}$ & [12] \\
\hline 4 & Polgar et al. & 2018 & Human & DNR-GnRH-based conjugates have no cytotoxic effect on cardiomyocytes & {$[13]$} \\
\hline 5 & Newman et al. & 1981 & Rat & $\begin{array}{l}\text { DNR myocardial toxicity can be prevented by adenosine }(10 \mu \mathrm{M} \text { to } 1 \mathrm{mM}) \text { for } 24 \mathrm{hr} \\
\text { prior to addition of DNR to myocardial cells }\end{array}$ & [14] \\
\hline 6 & Owattanpanich et al. & 2018 & Human & $\begin{array}{l}\text { The complete response rate after the first course of induction therapy was significantly } \\
\text { greater among adult patients with acute myeloid leukemia who had received idarubi- } \\
\text { cin as part of induction therapy compared with those who had received high dose of } \\
\text { DNR. But significant difference between cardiotoxicity rate of DNR and idarubicin } \\
\text { was not found }\end{array}$ & {$[15]$} \\
\hline 7 & Liu et al. & 2015 & Mice & $\begin{array}{l}\text { DNR - cardiac myosin interaction is the particular mode of inducing daunorubicin } \\
\text { cardiotoxicity }\end{array}$ & {$[16]$} \\
\hline 8 & AI-Kuraishy and AI-Gareeb. & 2016 & Rat & $\begin{array}{l}\text { Pomegranate demonstrated significant cardioprotection in DNR-induced cardiotoxic- } \\
\text { ity through reduction of oxidative stress, lipid peroxidation, inflammation, and cardiac } \\
\text { injury biomarkers }\end{array}$ & {$[17]$} \\
\hline 9 & Cusack et al. & 1997 & Rat & $\begin{array}{l}\text { There are significant age-related changes in DNR and daunorubicinol kinetics in the } \\
\text { rat that could alter susceptibility to acute systemic toxicity and to chronic cardiotoxic- } \\
\text { ity }\end{array}$ & {$[18]$} \\
\hline 10 & Maayah et $a l$. & 2018 & Rat and Human & $\begin{array}{l}\text { DNR induces cardiotoxicity through a sEH inhibitor-mediated cardioprotective EETs } \\
\text { degradation-dependent mechanism }\end{array}$ & [19] \\
\hline
\end{tabular}


$\mu \mathrm{M}$ produced significant release of creatine, phosphokinase and lactic dehydrogenase without a detectable decrease in cell viability. With pre-incubation of the myocardial cells with varying concentrations of adenosine $(10 \mu \mathrm{M}$ to $1 \mathrm{mM})$ for $24 \mathrm{hr}$ before the addition of anthracycline decreased or prevented drug-induced enzyme release, thus presenting adenosine as an effective myocardial protectant. It had no significant effect on cellular uptake of DNR, nor did adenosine adversely affect the oncolytic activity of DNR against L1210 leukemia cells. On the contrary, myocardial protectants including N-acetyl-Lcysteine, alpha-tocopherol and carnitine were found to be ineffective in preventing anthracycline-induced enzyme release [14].

Studies have been found which were conducted to compare the efficacy and toxicity of DNR with idarubicin. In one of the studies, 1809 participants met the eligibility criteria and were included in the metaanalysis. The patients treated with idarubicin presented a significantly greater complete response rate after the first course of induction therapy compared with those treated with high dose of DNR. Moreover, a significantly lower rate of refractory acute AML was observed in patients receiving idarubicin as compared to DNR [15]. The interaction between DNR and cardiac myosin can be one of the modes of cardiotoxicity. To investigate this fact, forty groups of mice were used in which mice were treated with DNR orally, and three DNR-treated groups in which mice were injected intraperitoneally with DNR at seven bolus doses of $2.0,4.0$, and $6.0 \mathrm{mg} / \mathrm{kg}$ body weight, respectively. Results revealed that weakly acidic environment ( $\mathrm{pH} 4.0-6.0)$ or higher temperature (30$37^{\circ} \mathrm{C}$ ) promoted the interaction between DNR and cardiac myosin, causing variations in conformation and normal physiological functions of cardiac myosin. Thermodynamic studies showed that the binding of DNR to cardiac myosin was a spontaneous process driven by entropy. It also indicated that hydrophobic interaction and hydrogen bonds may play essential roles in the combination of DNR with cardiac myosin. In addition, 4.0-6.0 mg/kg DNR-treated mice exhibited obvious increase in myocardial enzyme level, and reductions in blood cell count [16]. Oxidative stress and free radical formation are also involved in DNR cardiotoxicity. Pomegranate may play a significant role in scavenging free radical activity. To find the effect of pomegranate on DNR induced cardiotoxicity, 21 male sprague rats were divided into three groups out of which Group A received distilled water, Group B was treated with DNR $(20 \mathrm{mg} / \mathrm{kg}$ via intraperitoneal injection daily for 12 days for total cumulative dose of $240 \mathrm{mg} / \mathrm{kg}$ ) and Group C was the pretreatment group with pomegranate $(25 \mathrm{mg} / \mathrm{kg}$ for 6 days orally, then DNR $20 \mathrm{mg} / \mathrm{kg}$ administrated concomitantly for the next 6 days with a cumulative dose of $120 \mathrm{mg} / \mathrm{kg}$ ). Cardiac troponin I ([cTn I] pg/ml), malondialdehyde (MDA) (ng/ml), interleukin 17 (IL-17 pg/ml), and cardiac lactate dehydrogenase $(\mathrm{LDH})(\mathrm{pm} / \mathrm{ml})$, were used as biomarkers to measure the severity of cardiotoxicity. In group B, DNR induced lipid peroxidation and pro-inflammatory changes. In Group C, pomegranate pre-treatment demonstrated a significant cardio-protection in DNRinduced cardiotoxicity through reduction in oxidative stress, lipid peroxidation, pro-inflammatory, and cardiac injury biomarkers [17].

Significant age-related changes were noticed in DNR and daunorubicinol kinetics in the rats that may alter susceptibility to acute systemic toxicity and chronic cardiotoxicity. Systemic clearance of DNR was decreased in older rats in comparison to younger rats. Moreover, concentrations of DNR in plasma and heart were found to be higher in older as compared to younger rats [18]. DNR-induced cardiotoxicity was mediated through the induction of cardiotoxic hydroxyeicosatetraenoic acids and/or the inhibition of cardioprotctive epoxyeicosatrienoic acids (EETs). To investigate into it, Sprague-
Dawley rats were treated with DNR (5 mg/kg i.p.) for $24 \mathrm{~h}$, whereas human ventricular cardiomyocytes (RL-14) cells were exposed to DNR in the presence and absence of 4-[[trans-4-[[(tricyclo[3.3.1.13,7]dec1-ylamino)carbonyl]amino]cyclohexyl]oxy]-benzoic acid (tAUCB), a soluble epoxide hydrolase ( $\mathrm{sEH}$ ) inhibitor. This study provided the evidence that DNR induces cardiotoxicity through a $\mathrm{sEH}$-mediated EETs degradation-dependent mechanism [19]. Cardiotoxicity induced by DNR could be protected by sodium ferulate (SF). Forty juvenile Sprague Dawley (SD) rats were divided into four groups. $1^{\text {st }}$ group was taken as control group whereas $2^{\text {nd }}, 3$ rd and $4^{\text {th }}$ groups were treated with $\mathrm{DNR}, \mathrm{DNR}+\mathrm{SF}, \mathrm{SF}$ respectively. Juvenile rats were intraperitoneally treated with DNR $(2.5 \mathrm{mg} / \mathrm{kg}$ every week for a cumulative dose of $10 \mathrm{mg} /$ $\mathrm{kg}$ ) preparation immature myocardial injury model in presence with SF $(60 \mathrm{mg} / \mathrm{kg})$ oral treatment for 25 days. It was found that $S F$ could inhibit the decrease in heart rate induced by DNR $(\mathrm{p}<0.05)$. SF treatment also resulted in an increase in the left ventricular end diastolic pressure, heart rate, the maximal left ventricular systolic speed and the maximal left ventricular diastolic speed responding to isoproterenol stimulation ( $\mathrm{p}<0.01$ ); SF improved the myocardial ultrastructural injuries and inhibited the decline in cardiac Troponin I expression caused by DNR $(\mathrm{p}<0.05)[12]$.

\section{Conclusion}

Daunorubicin (DNR) has been reported with a significant role in treating leukemia but its use is restricted due to associated cardiotoxicity. DNR has been reported to induce sub-chronic cardiomyopathy as well as down regulation of cytokines and stem cell markers. DNR may affect the heart by forming hydrophobic interactions and hydrogen bonds with cardiac myosin. Other cardiotoxic effects may include inhibition of cardio-protective epoxyeicosatrienoic acid. In the field of amelioration, adenosine, sodium ferulate and pomegranate have been reported as capable cardio-protectants against DNR induced cardiotoxicity. Future research on amelioration of DNR associated toxicity is highly recommended so as to minimize the associated risk.

\section{Acknowledgement}

Authors thank head and staff of Department of Zoology, Khalsa College Amritsar for their constant support throughout the work.

\section{References}

1. Rizvi SFA, Tariq S, Mehdi M (2018) Anthracyclines: mechanism of action, classification, pharmacokinetics and future-A mini review. Int $J$ of Biotech \& Bioeng 4:81-85.

2. Nicholas R. Bachur (2010) Anthracyclines. In encyclopedia of cancer (Second Edition) 2002 .

3. Pescarmona G (2010) Anthracycline drugs.

4. Volkova M, Russell R (2011) Anthracycline cardiotoxicity: prevalence, pathogenesis and treatment. Curr Cardiol Rev 7: 214-220.

5. https://medlineplus.gov/druginfo/meds/a682289.html

6. https://www.oncolink.org/cancer-treatment/oncolink-rx/daunorubicin-cerubidine-r

7. http://chemocare.com/chemotherapy/drug-info/daunorubicin.aspx

8. Lubieniecka JM, Graham J, Heffner D, Mottus R, Reid R, et al. (2013) A discovery study of daunorubicin induced cardiotoxicity in a sample of acute myeloid leukemia patients prioritizes $\mathrm{P} 450$ oxidoreductase polymorphisms as a potential risk factor. Front Genet 4: 231. [Crossref]

9. Alloghbi AAS, Huff SB and Hamouda DM (2017) Fatal myocardial infarction secondary to daunorubicin in acute myeloid leukemia patient. $J$ Coron Heart Dis $1: 1$. 
10. Srankova J, Doka G, Pivackova L, Mesarosova L, Kyselovic J, et al. (2016) Daunorubicin Down-Regulates the Expression of Stem Cell Markers and Factors Involved in Stem Cell Migration and Homing in Rat Heart in Subchronic but not Acute Cardiomyopathy. Basic Clin Pharmacol Toxicol 119: 443-452. [Crossref]

11. Hoefer CC, Blair RH, Blanco JG (2016) Development of a CART Model to Predict the Synthesis of Cardiotoxic Daunorubicinol in Heart Tissue Samples from Donors with and Without Down Syndrome. J Pharm Sci 105: 2005-2008. [Crossref]

12. Lian JB, Wu ZJ, Fang QJ, Yu J, He RL (2015) Sodium ferulate protects against daunorubicin-induced cardiotoxicity in juvenile rats. 31: 54-8. [Crossref]

13. Polgar L, Lajko E, Soos P, Lang O, Manea M, et al. (2018) Drug targeting to decrease cardiotoxicity-determination of the cytotoxic effect of $\mathrm{GnRH}$-based conjugates containing doxorubicin, daunorubicin and methotrexate on human cardiomyocytes and endothelial cells. Beilstein J Org Chem 14:1583-1594.

14. Newman RA, Hacker MP, Krakoff IH (1981) Amelioration of adriamycin and daunorubicin myocardial toxicity by adenosine. Cancer Res 41: 3483-3488. [Crossref]
15. Owattanapanich W, Owattanapanich N, Kungwankiattichai S, Ungprasert P, Ruchutrakool T: Efficacy and toxicity of idarubicin versus high-dose daunorubicin for induction chemotherapy in adult acute myeloid leukemia: A systematic review and meta-analysis. Clin Lymphoma Myeloma Leuk 2018;18(12):814-821.

16. Liu Y, Chen C, Duan X, Ma W, Wang M, Tu M, Chen Y: Fluorescence spectra of cardiac myosin and in vivo experiment: studies on daunorubicin-induced cardiotoxicity. Iran J Basic Med Sci 2015;18(12):1199-208.

17. AI-Kuraishy HM, AI-Gareeb AI (2016) Potential effects of pomegranate on lipid proinflammatory changes in daunorubicin-induced cardiotoxicity in rats. Int J Prev Med 7: 85 .

18. Cusack BJ, Young SP, Vestal RE, Olson RD (1997) Age-related pharmacokinetics of daunorubicin and daunorubicinol following intravenous bolus daunorubicin administration in the rat. Cancer Chemother Pharmacol 39: 505-512. [Crossref]

19. Maayah ZH, Abdelhamid G, Elshenawy OH, El-Sherbeni AA, Althurwi HN, et al. (2018) The Role of Soluble Epoxide Hydrolase Enzyme on Daunorubicin-Mediated Cardiotoxicity. Cardiovasc Toxicol 18: 268-283. [Crossref]

Copyright: $@ 2019$ Mendelson M. This is an open-access article distributed under the terms of the Creative Commons Attribution License, which permits unrestricted use, distribution, and reproduction in any medium, provided the original author and source are credited. 